\title{
Os indicadores de qualidade da prescrição de medicamentos da Organização Mundial da Saúde ainda são válidos?
}

\author{
Are the drug prescription quality indicators \\ of the World Health Organization still valid?
}

Samanta Etges Fröhlich ${ }^{1}$

Sotero Serrate M engue ${ }^{1}$

${ }^{1}$ UniversidadeFederal do

Abstract The scope of this article is to analyze the drug prescription quality indicators proposed by the World Health Organization in 1993 and suggest new indicators attuned to the new profile of illness of the population. For this purpose, a cross-sectional study was conducted by means of personal interviews and prescription analysis among 320 patients who were attended at Strategic Family Health U nits located in a Brazilian municipality in the state of Rio Grande do Sul. The users were recruited by the consecutive nonprobabilistic samplingmethod. In accordance with the drug prescription quality indicators of the World $\mathrm{H}$ ealth Organization, the prescriptions can beconsidered adequate. However, analysis of those indicators shows that they may currently be inappropriate. Therefore, new indicators were cre ated. Using the new, easily quantifiable and straightforward indicators proposed, it was possible to detect the problems in a drug prescription with a view to taking corrective action. Key words Prescription, Quality of drug prescription indicators, Primary Healthcare
Resumo Este artigo tem como objetivo analisar os indicadores de qualidadeda prescrição demedicamentos propostos em 1993 pela Organização M undial da Saúdee sugerir novosindicadores, em razão do novo perfil de morbidade da população. Para tanto, serealizou um estudo transversal, através de entrevista pessoal e análise de prescrições de 320 usuários de U nidades de Estratégia Saúde da Família em um município brasileiro no estado do Rio Grande do Sul. Esses usuários foram recrutados por meio do método de amostragem não probabilístico consecutivo. Considerando os resultados obtidos com os indicadores de qualidade da prescrição propostos pela O rganização M undial da Saúde, as prescrições podem ser consideradas adequadas. Contudo, a análisedesses indicadores mostrou que, atualmente, eles podem ser inapropriados. Portanto, novos indicadores foram desenvolvidos. Pela nova proposta deindicadores, simplese de fácil quantificação, pôde-se perceber os problemas em uma prescrição de medicamentos com 0 intuito de guiar para uma ação subsequente. Palavras-chave Prescrição, Indicadores de qualidade da prescrição, Atenção Primária 
Introdução

Qualidadeé um passo no qual o serviço de saúde conhece as necessidades, as expectativas e o padrão de saúde dos pacientes, de seus familiares e deoutros beneficiários ${ }^{1}$. Para se chegar à qualidade, são necessários planejamento everificação das falhas, feitas através da análise dos serviços e de registros, como a prescrição de medicamentos.

Indicadores podem ser utilizados para avaliar a qualidade de uma prescrição. Eles são medidas qualitativas ou quantitativas que avaliam desempenhos, detectam problemas e orientam a solução. Podem ser empregados em qualquer aspecto da cadeia de assistência: pessoas, processos, equipamentos ou instituições de saúde?2.

Os indicadores de qualidade da prescrição de medicamentos preconizados pela Organização Mundial da Saúde (OMS) ${ }^{3}$ foram e ainda são utilizados para avaliar qualidade. Eles incluem 0 número médio de medicamento por prescrição, a porcentagem de medicamentos prescritos pela denominação genérica, a porcentagem de prescrições com pelo menos um antibiótico, a porcentagem de prescrição com pelo menos um medicamento injetável ea porcentagem demedicamentos prescritos que fazem parte da Lista de M edicamentos Essenciai $s^{3}$. Essesindicadores são antigos e não sabemos se são válidos no contexto atual.

Segundo a $\mathrm{OMS}^{3}$, o número médio de medicamento por prescrição tem o propósito de verificar o grau de polifarmácia, que, em alguns casos, é necessária. Pesquisas mostraram que se 0 número de medicamentos for maior que cinco, há maior risco de eventos adversos relacionados aos medicamentos $s^{4,5}$. A idade da população deve ser levada em conta, uma vez que a tendência é aumentar o número de medicamentos por prescrição quanto mais idosa é a população (pois maior é o número de morbidades).

A porcentagem de medicamentos prescritos pelo nome genérico visa medir a tendência do local para a prescrição de genéricos. A Política de M edicamentos Genéricos foi implantada nos últimos anos no Brasil e sua efetivação está relacionada à adesão dos prescritores, sendo a denominação genérica um fator importante na promoção do uso racional do medicamento, como também na redução de custos com medicação ${ }^{2,3,6,7}$. É a Lei $n^{\circ} 9.787^{8}$ que define o que é 0 medicamento genérico e como ele deverá superar o medicamento de referência.

A porcentagem de prescrições com pelo menosum antibiótico nos diz quais são os antibióti- cos mais prescritos e a cultura da população estudada em relação ao antibiótico ${ }^{3,6}$. 0 principal problema quanto à prescrição de antibióticosé o seu uso abusivo, que gera o desenvolvimento de microrganismos potencialmente resistentes a qualquer tratamento, acarretando graves consequências ao paciente, podendo levar ao óbito ${ }^{9,10}$.

A porcentagem de prescrições com pelo menos um medicamento injetável mede o seu grau de utilização. Com este parâmetro, pode-se perceber a cultura de prescritores e pacientes no que diz respeito a esta forma farmacêutica ${ }^{3,6,9}$.

Os medicamentos essenciais surgiram como meio de possibilitar o acesso aos medicamentos a um maior número de pessoas, e sua adoção se baseia na segurança, eficácia terapêutica comprovada, qualidade e disponibilidade. Essa iniciativa alcançou adesão maior que a esperada, com fármacos de eficácia garantida e comprovada e com baixo índice risco/benefício ${ }^{2,11}$. D aí a importância de se verificar o grau de conformidade do local com a política nacional de medicamentos, de modo a se avaliar se a Lista de Medicamentos Essenciais vai ao encontro da necessidade da população ${ }^{3,6}$.

A partir da análise desses indicadores de qualidade da prescrição, pretende-se propor novos indicadores baseados nos problemas encontrados nas prescrições avaliadas. Além disso, pretende-se comparar os indicadores da OM S com a opinião do paciente, pois a perspectiva do paciente traz sua opinião, necessidades, experiências epercepções.

\section{Métodos}

Esta pesquisa seguiu um model o de estudo transversal e tem como alvo indivíduos cadastrados nas Unidades de Estratégia Saúde da Família (ESF) do município de Santa Cruz do Sul (RS, Brasil).

Para serem incluídas no estudo, as pessoas deveriam ser cadastradas nas Unidades de Estratégia Saúde da Família, ter acima de 18 anos de idade, aceitar fazer parte do estudo, ser capaz de se comunicar adequadamente, ter utilizado os serviços médicos da ESF entre o período da coleta de dados (março a maio de 2006) e ter uma prescrição médica a cumprir.

Por meio de uma amostragem consecutiva, foram entrevistados todos os usuários que atendiam aos critérios de inclusão e que se encontravam disponíveis para a entrevista após o término da consulta. 
Os entrevistados assinaram um Termo de Consentimento Livre e Esclarecido, no qual garantia-se a privacidade dos participantes, explicava-se a finalidade do estudo e que a não participação não afetaria o atendimento na Unidade em questão, além do meio de contato para esclarecimento de qualquer dúvida.

A entrevista foi o método escolhido para coletar dados sociodemográficos e opiniões dos usuários. As prescrições de medicamentos dos usuários foram transcritas e analisadas por um farmacêutico para avaliar a sua qualidade com relação aos indicadores da OM S e aos indicadores propostos.

Os dados tiveram dupla digitação por meio do programa Epilnfo versão 3.3.2. As análises estatísticas realizadas foram descritivas e efetuadas com o programa SPSS versão 13.0. Os medicamentos foram classificados de acordo com a Anatomical Therapeutic Chemical (ATC) ${ }^{12}$.

A pós a análise das prescrições de medicamentos, novos indicadores de qualidade da prescrição foram sugeridos como resultado deste estudo.

Para a comparação dos indicadores propostos com os estabelecidos pela OMS e verificar suas diferenças, realizou-se a seguinte classificação: considerando-se que cada situação ideal de cada indicador represente 1 ponto, tem-se uma pontuação de 0 a 5 para os indicadores da OM S e, para os indicadores propostos, uma pontuação de 0 a 7 - pois são 7 indicadores. Assim, classificaram-se como prescrições de baixa qualidade as prescrições com pontuação de 0 a 3 pontos para os indicadores da OMS e de 0 a 4 pontos para os novos indicadores. As demais prescrições foram consideradas de alta qualidade. Com esta classificação, foi possível comparar os indicadores e verificar as suas diferenças.

0 projeto para a execução deste estudo foi aprovado pela Secretaria da Saúde do município deSanta Cruz do Sul (RS) e pelo Comitê de Ética da Universidade Federal do Rio Grande do Sul.

\section{Resultados}

Participaram do estudo 320 usuários das unidades de ESF. A Tabela 1 mostra as características sociodemográficas da amostra estudada.

A Tabela 2 mostra os resultados dos indicadores de qualidade da prescrição da OMS e a opinião dos usuários com relação a esses indicadores. Procurou-se saber a opinião dos usuários sobre qual o número de medicamentos que 0 entrevistado acha ideal um médico prescrever, se aprova ou não quando o médico receita medicamento pela denominação genérica, segosta quando o médico receita antibiótico, se o entrevistado acha interessante o médico receitar um medicamento na forma injetável e se ele acha necessário que o medicamento prescrito esteja disponível na Farmácia Básica Municipal.

No modelo proposto de indicadores de qualidade da prescrição, foram mantidos os seguintes indicadores da OM S (em razão da sua importância): medicamentos prescritos pela denominação genérica, porcentagem de prescrições contendo pelo menos um antibiótico e número de medicamentos prescritos que constam na Lista de M edicamentos Essenciais. Além destes, com base nas prescrições da amostra estudada, desenvolveram-se os seguintes indicadores:

- Proporção de prescrições contendo mais de um medicamento da mesma classe farmacológica, cujo resultado foi de 9,0\% (29);

- Proporção de prescrições contendo medicamento na forma de soluções orais para pacientes

Tabela 1. Características sociodemográficas da população estudada.

\begin{tabular}{|c|c|c|}
\hline Variáveis & $\mathrm{N}$ & $\%$ \\
\hline \multicolumn{3}{|l|}{ Sexo } \\
\hline M asculino & 95 & 29,7 \\
\hline Feminino & 225 & 70,3 \\
\hline \multicolumn{3}{|l|}{ Cor } \\
\hline Branca & 193 & 60,4 \\
\hline Preta & 51 & 15,9 \\
\hline Amarela & 8 & 2,5 \\
\hline Parda & 67 & 20,9 \\
\hline Indígena & 1 & 0,3 \\
\hline \multicolumn{3}{|l|}{ Situação conjugal } \\
\hline $\begin{array}{l}\text { Vive com cônjuge ou } \\
\text { companheiro(a) }\end{array}$ & 244 & 76,3 \\
\hline $\begin{array}{l}\text { Não vive com o cônjuge ou } \\
\text { companheiro(a) }\end{array}$ & 76 & 23,7 \\
\hline \multicolumn{3}{|l|}{ Escolaridade } \\
\hline Nunca frequentou & 24 & 7,5 \\
\hline $1^{\circ} \mathrm{grau}$ & 242 & 75,6 \\
\hline $2^{\circ} \mathrm{grau}$ & 47 & 14,7 \\
\hline $3^{\circ} \mathrm{grau}$ & 7 & 2,2 \\
\hline \multicolumn{3}{|l|}{ Renda mensal } \\
\hline Até $\mathrm{R} \$ 300,00$ & 134 & 41,9 \\
\hline De $R \$ 301,00$ a $R \$ 600,00$ & 141 & 44,0 \\
\hline Acima de $\mathrm{R} \$ 600,00$ & 45 & 14,1 \\
\hline \multicolumn{3}{|l|}{ Idade } \\
\hline De 18 a 40 anos & 131 & 41,0 \\
\hline De 41 a 60 anos & 132 & 41,2 \\
\hline Acima de 60 anos & 57 & 17,8 \\
\hline
\end{tabular}


adultos, em queestá disponível a forma farmacêutica sólida, tendo como resultado $11,6 \%$ (37);

- Número de psicotrópicos prescritos. $\mathrm{Na}$ amostra avaliada, 9,2\% (54) dos medicamentos prescritos eram psicotrópicos e 14,3\% (46) das prescrições continham algum psicotrópico prescrito;

- A prescrição contém as informações mínimas necessárias para o uso do medicamento, que são: nome, dose, posologia e tempo de uso dos medicamentos prescritos - esta última informação só não é fundamental para os pacientes com doenças crônicas. $62,8 \%$ (201) das prescrições continham as informações consideradas mínimas necessárias para o uso do medicamento.

O Gráfico 1 mostra os indicadores propostos para diferentes faixas de idade dos usuários.

Quanto ao gênero, a proporção das prescrições das mulheres com medicamentos psicotrópi$\cos (16,3 \%)$ foi maior do quea doshomens $(9,5 \%)$, com um valor de $p=0,02$ no qui-quadrado.

As diferenças entre os demais indicadores e as características sociodemográficas não foram significativas, isto é, com valor de $p>0,05$.

Os resultados da comparação entre os dois grupos de indicadores encontram-se na Tabela 3. Observou-se que em $33,5 \%$ das prescrições foram verificadas diferenças de classificação entre os grupos de indicadores comparados com relação a alta e baixa qualidade. $23,2 \%$ das prescri-

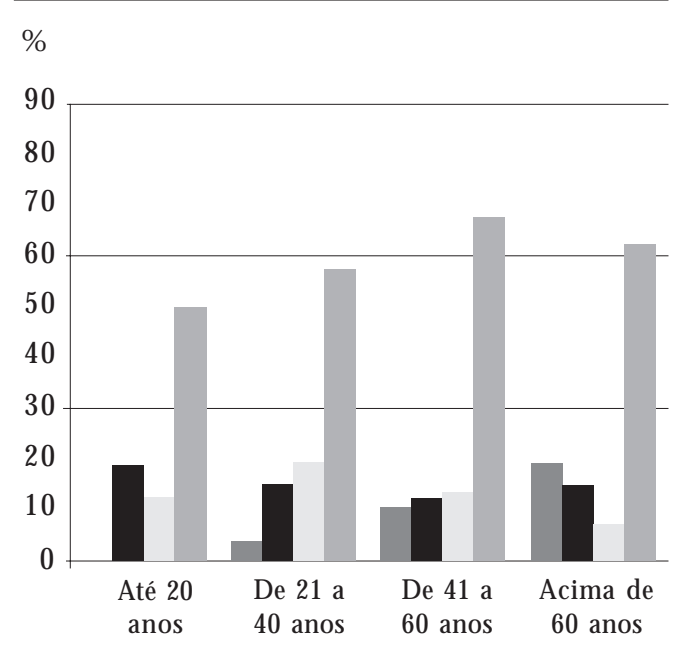

Proporção de prescrições contendo medicamentos da mesma classe farmacológica

- Proporção de prescrições contendo soluções orais

Proporção de medicamentos psicotrópicos prescritos

Proporção de prescrições com as informações mínimas necessárias para o uso do medicamento

Gráfico 1. Indicadores de qualidade da prescrição propostos para diferentes faixas de idade dos usuários.

Tabela 2. Indicadores da OM S com base nas prescrições e segundo a opinião dos usuários das unidades de ESF de Santa Cruz do Sul (RS).

\begin{tabular}{lcc}
\hline \multicolumn{1}{c}{ Indicadores da OMS } & $\begin{array}{c}\text { Conteúdo das } \\
\text { prescrições }\end{array}$ & $\begin{array}{c}\text { Opinião dos } \\
\text { usuários }\end{array}$ \\
\hline Número de medicamentos por prescrição & 1,8 & 1,7 \\
Proporção de medicamentos prescritos pela denominação genérica & $84 \%$ & $80 \%$ \\
Proporção de prescrições contendo antibióticos & $8 \%$ & $56 \%$ \\
Proporção de prescrições contendo injetáveis & $3 \%$ & $63 \%$ \\
Proporção de medicamentos prescritos que constam na Lista de & $81 \%$ & $93 \%$ \\
Medicamentos Essenciais & &
\end{tabular}

Tabela 3. Comparação da classificação entre alta e baixa qualidade entre os indicadores da OM S e oS indicadores propostos.

\begin{tabular}{lccr}
\hline & \multicolumn{2}{c}{ Indicadores OMS } \\
\cline { 2 - 3 } \multicolumn{1}{c}{ Indicadores propostos } & Alta qualidade (\%) & Baixa qualidade (\%) & Total \\
\hline Alta qualidade (\%) & 89,7 & 10,3 & \\
Baixa qualidade (\%) & 23,2 & 76,8 & 100,0 \\
Total & & & 100,0 \\
\hline
\end{tabular}


ções foram classificadas como de alta qualidade pela OM S e de baixa qualidade pelos novos indicadores; além disso, 10,3\% das prescrições foram classificadas como de baixa qualidade pela OMS e de al ta qualidade pelos novos indicadores.

\section{Discussão}

Pelas características sociodemográficas da amostra estudada, percebe-se que ela é composta, em sua maioria de mulheres, com baixa escolaridade ebaixo poder socioeconômico.

O número médio demedicamentos por prescrição foi considerado bom pela $\mathrm{OMS}^{3}$. Se comparado com outros estudos realizados, este número está abaixo deles - entre 2 e 2,5 medicamentos por prescrição $0^{6,9,13,14}$.

0 uso da denominação genérica foi alto se comparado às demais pesquisas - entre $28,4 \mathrm{e}$ $73,3^{6,9,13}$. A pesar deste alto número, cabe ressaltar que a legislação brasileira obriga o uso de denominação genérica em todas as prescrições no âmbito do SUS .

A baixa indicação de antibióticos pode estar relacionada ao período do ano em que foi realizada a coleta de dados (março-maio), mas também pode-se pressupor maior preocupação dos prescritores em não prescrevê-los para situações em que não são necessários. Como a falta crônica desses medicamentos não foi mencionada pelos usuários, a baixa indicação não se devea baixa disponibilidade.

Os medicamentos na forma de injetáveis podem ser considerados em desuso na amostra estudada, talvez em razão de a Lista de M edicamentos Essenciais do município restringir esta forma farmacêutica, e ao fato de as unidades só disponibilizarem injetáveis para procedimentos no posto e não para retiradas. Em outros estudos similares, a porcentagem de injetáveis prescritos variou entre 7,5\% e 8,3\% ${ }^{9,13}$.

A Lista de M edicamentos Essenciais do município podeser considerada aceita pel os prescritores locais, visto o alto número de prescrições com medicamentos que fazem parte dela.

Através dos resultados dos indicadores de qualidade da prescrição definidos pela OMS, as prescrição analisadas são de qualidade, uma vez que estudos demonstraram que o número mé dio de medicamentos por prescrição fica em torno de 1,3 a 2,2; a porcentagem de genéricos prescritos fica entre $82 \%$ e $94 \%$; a porcentagem de antibiótico fica entre $29 \%$ e $43 \%$; a porcentagem deinjetáveis fica entre $0,2 \%$ e $48 \%{ }^{3}$.
Os eventuais problemas das prescrições podem não ser detectados pelos indicadores da OMS em razão do novo perfil de morbidade da população.

$\mathrm{N}$ as questões relacionadas ao número de medicamentos por prescrição e ao uso de denominação genérica, as opiniões dos entrevistados são similares ao encontrado nas prescrições avaliadas, revelando que os indivíduos consideram ideal um médico prescrever poucos medicamentos, além da aceitação dos genéricos pela população. M as com relação aos antibióticos, observou-se uma grande diferença através da Tabela 2 , revelando que as pessoas não mostram restrição ao uso de antibióticos, talvez pelo imaginário da população sobre o poder quase mágico desses medicamentos. Além disso, em alguns casos, os entrevistados podem não saber o que é um antibiótico ou até confundi-lo com outras classes de medicamentos. 0 mesmo ocorreu com os injetáveis, pois, apesar de estarem em desuso, eles pareceram ser bem aceitos pela população estudada. Com relação à Lista de M edicamentos Essenciais, os entrevistados desejam que seus medicamentos estejam contidos nela.

Os dados relacionados à opinião dos usuários mostram as necessidades, experiências e percepções deles - isto não significa que os usuários têm que receber necessariamente 0 que desejam em termos de medicamentos - , e essa percepção pode ser um balizador no momento de avaliar as expectativas dos pacientes. Assim, antibióticos e injetáveis parecem criar nos usuários a sensação de que estão sendo usados medicamentos mais fortes, com isso a doença está sendo mais atacada e a cura deve ser mais rápida. Esses medicamentos trazem um imaginário de maior efetividade, enquanto as avaliações técnicas reservam esses medicamentos para usos mais restritos.

Asprescrições contendo mais de um medicamento da mesma classe farmacológica podem ser potencialmenteprejudiciais, com maior risco de interações e de medicamentos desnecessários. Essas prescrições também podem dificultar a adesão, já que se o número de medicamentos utilizadosérelativamente pequeno, os indivíduos são mais propensos a fazer melhor uso deles e realizam mudanças na prescrição com menor frequência que os demais $5^{5,15}$.

A pesar do fato de que as intervenções devem priorizar aumento da qualidade na prescrição de medicamentos, não se deve descartar o custo das ações em saúde, e esse novo indicador mostrou que nessas prescrições há maior custo do medicamento para a sociedade $e^{5,16}$. 
As prescrições contendo medicamentos na forma de soluções orais não são as ideais para pacientes adultos, devido à menor estabilidade desta forma farmacêutica quando comparada com a forma farmacêutica sólida. A forma farmacêutica líquida está mais sujeita à decomposição por hidrólise - quando se utiliza como véculo a água -, oxidação - fármaco mantido em outro estado que não o sólido, pela presença de oxigênio, além da exposição à luz - econtaminação microbiana ${ }^{17}$.

O número de medicamentos psicotrópicos prescritos foi proposto devido ao uso indiscriminado e cada vez mais constante de pessoas que os utilizam - especialmente os benzodiazepínicos. Pelos resultados, verifica-se maior prevalência na amostra estudada de prescrições associadas ao tratamento de doenças relacionadas ao sistema nervoso.

Com relação ao último indicador proposto, esperava-se que todas as prescrições viessem acompanhadas das informações mínimas necessários, o que não ocorreu.

Pela Figura 1, observa-se uma relação linear de crescimento quanto à idade e ao número de medicamentos da mesma classe farmacológica, que pode ser explicado pelo aumento da morbidade com o passar dos anos, o que, muitas vezes, exige a terapêutica múltipla de medicamentos. Com relação aos medicamentos psicotrópicos, a faixa etária em que são mais prescritos é entre 21 e 40 anos, mostrando o perfil da população jovem atual, que é de pessoas com alto índice de estresse e aumento do risco de ansieda$\mathrm{de}^{18}$. Segundo $\mathrm{H}$ enderson et al. ${ }^{19}$, a ansiedade e a depressão declinam com a idad;, além disso, os transtornos psiquiátricos e, por consequência, a utilização demedicamentos controlados são maiores em adultos jovens. Os medicamentos prescritos na forma de solução oral e as prescrições contendo as informações mínimas para a utilização segura dos medicamentos podem ser considerados sem mudanças significativas com 0 aumento da idade.

0 fato das mulheres receberem prescrição de medicamentos psicotrópicos com maior frequência pode ser explicado por elas possuírem um risco significativamente maior do que os homens de desenvolver transtorno de ansiedade, depres- são e desordens afetivas sazonais, mas há poucos estudos nesta área, sugerindo os resultados preliminares que fatores genéticos (hormônios sexuais eseus ciclos) eambientais possam estar envolvidos no desenvolvimento da ansiedade ${ }^{18,20,21}$.

Pela comparação dos dois grupos de indicadores, verificou-sequeeles classificaram duas em cada três prescrições igualmente - alta e baixa qualidade. Este resultado deve-se à manutenção de três indicadores da OM S nos novosindicadores propostos. Uma em cada três prescrições foram classificadas de forma diferente. As prescrições que foram consideradas de boa qualidade pela OMS, mas não pelos novos indicadores, se referem especialmente às prescrições contendo medicamentos da mesma classe farmacológica, presença de soluções orais e psicotrópicos, além da ausência das informações mínimas para o uso correto do medicamento - informações estas que os indicadores da OMS não consideram. Já as prescrições consideradas de baixa qualidade pela OMS e de alta qualidade pelos novos indicadores se devem pela consideração do número de medicamentos prescritos pela OMS.

Algumas prescrições analisadas devem ser repensadas a fim de eliminar a presença de medicamentos desnecessários, levando em conta o custo-benefício e promovendo a segurança dos usuários.

Com o desenvolvimento das novas ferramentas, simples e de fácil quantificação na prática clínica, pôde-se perceber problemas nas prescrições que passam despercebidos com a utilização dos indicadores de qualidade da prescrição da OMS. Dessa forma, os indicadores propostos são simples e possibilitam acompanhar as práticas de prescrição de medicamentos sem que isso de mande o exercício de treinamentos complexos e sem que haja a necessi dade de aplicação de grandes recursos para a pesquisa de tais indicadores.

Os indicadores propostos são entendidos como medidas de primeira linha; afinal, qualidade não pode ser medida somente através de um indicador. A intenção éestimular outrosquestionamentos e guiar para uma ação subsequente. Além disso, como os indicadores são desenvolvidos como medidas estatísticas quantitativas, é importante continuar na investigação de les, com o intuito de aperfeiçoá-los e validá-los. 


\section{Colaboradores}

SE Fröhlich trabalhou na concepção da pesquisa, na metodologia, análise, interpretação e redação do artigo; SS M engue, no delineamento, metodologia e revisão crítica do artigo.

\section{Referências}

1. Donabedian A. Twenty years of research on the quality of medical care: 1964-1984. Eval Health Prof 1985; 8(3):243-265.

2. Castro CGSO. Estudos de utilização de medicamentos: noções básicas. 1a reimpr. Rio de Janeiro: Editora Fiocruz; 2002.

3. World Health Organization (WHO). How to investigate drug use in health facilities. Geneva: WHO; 1993. [serial on the Internet]. [cited $2005 \mathrm{M}$ ay 30]. Avaible from: http://www.who.int/medicines/library/theme/ theme_res.shtml

4. Odubanjo E, Bennett K, Feely J. Influence of socioeconomic status on the quality of prescribing in the elderly: a population based study. Br J Clin Pharmacol $2004 ; 58(5): 496-502$.

5. Roth MT, Weinberger M, Campbell WH. Measuring the quality of medication use in older adults. J Am Geriatr Soc 2009; 57(6):1096-1102.

6. Acurcio FA, Perini E, Magalhaes SM, Terceiro LG, Vieira Filho JM, Coutinho KE, , Caldeira KV, Cabral LH, Santos MC, Arantes PM, Vale SA, Souza MC. Analysis of medical prescriptions dispensed at health centers in Belo Horizonte, Minas Gerais, Brazil. Cad Saude Publica 2004; 20(1):72-79.

7. Campbell SM, Roland MO, Buetow SA. Defining quality of care. Soc Sci M ed 2000; 51(11):1611-1625.

8. Brasil. Lei $n^{\circ}$ 9.787, de 10 de fevereiro de 1999. Altera a Lei no 6.360, de 23 de setembro de 1976, que dispõe sobre a Vigilância Sanitária, estabelece o medicamento genérico, dispõe sobre a utilização de nomes genéricos em produtos farmacêuticos e dá outras providências. 1999; 10 fev [acessado 2006 jul 27]. Disponível em: http://www.anvisa.gov.br/ legis/leis/9787_99.htm

9. Santos V, Nitrini SM. Prescription and patient-care indicators in healthcare services. Rev Saude Publica 2004; 38(6):819-826.

10. Schenkel EP. Cuidados com os medicamentos. $3^{\mathrm{a}}$ ed. Porto Alegre: Universidade Federal do Rio Grande do Sul; Florianópolis: Universidade Federal de Santa Catarina; 1996.

11. Brasil. Portaria no 1.587, de 3 de setembro de 2002. Aprova a revisão da Relação Nacional de Medicamentos Essenciais - Rename. Diário Oficial da União 2002; 3 set. [acessado 2005 jun 6]. Disponível em: http://www.anvisa.gov.br/medicamentos/essencial. htm

12. World Health Organization (WHO). Collaborating Centre for Drug Statistics M ethodology. Anatomical Therapeutic Chemical classification system with Defined Daily Doses (ATC/DDD Index), 2006. [serial on the Internet] [cited 2006 Jul 4]. Available from: http://www.whocc.no/atcddd/

13. Naves JO, Silver LD. Evaluation of pharmaceutical assistance in Public Primary Care in Brasilia, Brazil. Rev Saude Publica 2005; 39(2):223-230.

14. Otoom S, Batieha A, Hadidi H, Hasan M, Al-Saudi K. Evaluation of drug use in Jordan using WHO patient care and health facility indicators. East M editerr Health J 2002; 8(4-5):544-549. 
15. Robertson J, Fryer JL, O'Connell DL, Smith AJ, Henry DA. Personal formularies: an index of prescribing quality? Eur J Clin Pharmacol 2001; 57(4):333-341.

16. Amado GE, Madridejos MR, Perez RM, Puig $X$. Relationship between quality and cost of the drug prescription in primary care. Aten Primaria 2000; 25(7):464-468.

17. Ansel HC, Popovich NG, Allen Jr LV. Desenvolvimento farmacotécnico: considerações gerais, matérias-primas e boas práticas de fabricação. In: Ansel HC, Popovich NG, Allen Jr LV. Farmacotécnica: formas farmacêuticas $\&$ sistemas de liberação de fármacos. $6^{a}$ ed. São Paulo: Editorial Premier; 2000. p. 113-173.

18. Gorman JM. Gender differences in depression and response to psychotropic medication. Gend M ed 2006; 3(2):93-109.

19. Henderson $A S$, Jorm $A F$, Korten $A E$, Jacomb $P$, Christensen $H$, Rodgers $B$. Symptoms of depression and anxiety during adult life: evidence for a decline in prevalence with age. Psychol M ed 1998; 28(6):13211328.

20. Azpiazu GM, Cruz JA, Villagrasa FJ, Abanades HJC, Garcia MN, Álvarez DM RC. Quality of life in noninstitutionalized persons older than 65 years in two health care districts in Madrid. Aten Primaria 2003; 31(5):285-292.

21. Kinrys G, Wygant LE. Anxiety disorders in women: does gender matter to treatment? Rev Bras Psiquiatr 2005; 27(Suppl.2):S43-S50.

Artigo apresentado em 29/01/2008 Aprovado em 19/08/2008

Versão final apresentada em 10/09/2008 